\title{
Vulnerabilidad a la contaminación de aguas subterráneas en sistemas de abastecimiento comunitario de Encarnación - Paraguay
}

\author{
María Rosa Servín Nasich \\ sernasich@gmail.com \\ Escuela de Postgrado. Universidad Nacional de Itapúa \\ Encarnación - Paraguay \\ Amado Insfrán Ortíz \\ amado.insfran@gmail.com \\ Grupo de Ecología y Restauración Forestal. Universidad de Alcalá \\ Alcalá de Henares - España
}

\section{RESUMEN}

Este estudio fue desarrollado en el distrito de Encarnación (Paraguay), y planteó dos objetivos: primero, distinguir los pozos de captación de aguas subterráneas para distribución en redes de tubería ubicados en las zonas de mayor vulnerabilidad a la contaminación, según el método DRASTIC; y, segundo, estimar el volumen de agua distribuida en estas redes que podría verse comprometido por la vulnerabilidad propia del acuífero, de acuerdo a un cálculo de extracciones. Así, se halló que gran parte de la población urbana se encuentra asentada en las áreas más vulnerables a la contaminación. Además, se estimó el volumen de aguas subterráneas que son distribuidas en redes de tubería, contabilizando un total de $0,004 \mathrm{~km}^{3} / a$ ño. Dada la alta dependencia de las aguas subterráneas de la población local, estos resultados pueden considerarse una señal de alerta ante el peligro potencial a la contaminación por las actividades económicas que se desarrollan en el distrito y las potenciales consecuencias sobre la salud pública. Los resultados obtenidos en este estudio pueden considerarse el primer paso hacia una gestión sostenible del recurso subterráneo. Así, el siguiente paso sería hacer un inventario de fuentes contaminantes y evaluar perímetros de protección de pozos, de acuerdo a las normativas vigentes.

Palabras clave: sistema acuífero guaraní; pozos de captación; volumen de extracción; peligro de contaminación 


\title{
Vulnerability to groundwater pollution in community supply systems of Encarnación - Paraguay
}

\begin{abstract}
This study was carried out in the district of Encarnación (Paraguay), and set two objectives: first, to distinguish the wells for groundwater distribution in pipe networks located in the areas of greatest vulnerability to contamination, according to the DRASTIC method; and, second, to estimate the volume of water distributed in these networks that could be compromised by the vulnerability of the aquifer, according to a calculation of withdrawals. Thus, it was found that a large part of the urban population is settled in the areas most vulnerable to contamination. In addition, the volume of groundwater that is distributed in pipe networks was estimated, accounting for a total of $0,004 \mathrm{~km}^{3} /$ year. Given the high dependence of the local population on groundwater, these results can be considered a warning sign against the potential danger of contamination due to the economic activities carried out in the district and the potential consequences on public health. The results obtained in this study can be considered the first step towards a sustainable management of the underground resource. Thus, the next step would be to make an inventory of polluting sources and evaluate well protection perimeters, in accordance with current regulations.
\end{abstract}

Keywords: guaraní aquifer system; catchment wells; volume of extraction; contamination risk.

Artículo recibido: 02 noviembre. 2021 Aceptado para publicación: 28 noviembre 2021

Correspondencia: sernasich@gmail.com Conflictos de Interés: Ninguna que declarar 


\section{INTRODUCCIÓN}

América Latina es la región del mundo que cuenta con la más alta disponibilidad per cápita de agua dulce (Fernández Colón 2009). Allí pueden encontrarse alrededor de 230.000 cuencas (Ruiz 2020), como la del Río de la Plata; además de 67 reservorios subterráneos transfronterizos (da Franca Ribeiro dos Anjos et al. 2007), como el Acuífero Guaraní (Fernáncez Cirelli y du Mortier 2005). Este último es considerado la reserva más grande de agua dulce transnacional, y es compartida por Brasil, Argentina, Paraguay y Uruguay (Wilkinson 2010).

Paraguay se encuentra entre los 10 países latinoamericanos con mayores reservas de agua dulce (Maboggiano 2019). La disponibilidad de agua es de aproximadamente 60.000 $\mathrm{m}^{3}$ /per cápita/año, considerada una de las más altas del mundo (PNUD 2006), resultado del informe DBEnvironment de 1992. Sin embargo, autores como Monte Domecq (2003) y Álvarez Enciso (2014), consideran que la cifra es más cercana a los $18.000 \mathrm{~m}^{3} / \mathrm{año}$ per cápita, tomando en cuenta que la disponibilidad hídrica tiende a disminuir como consecuencia natural del aumento demográfico. En la siguiente imagen se pueden visualizar los principales cauces como parte de los sistemas hidrológicos del país:

\section{Figura 1}

Sistemas hidrológicos de Paraguay

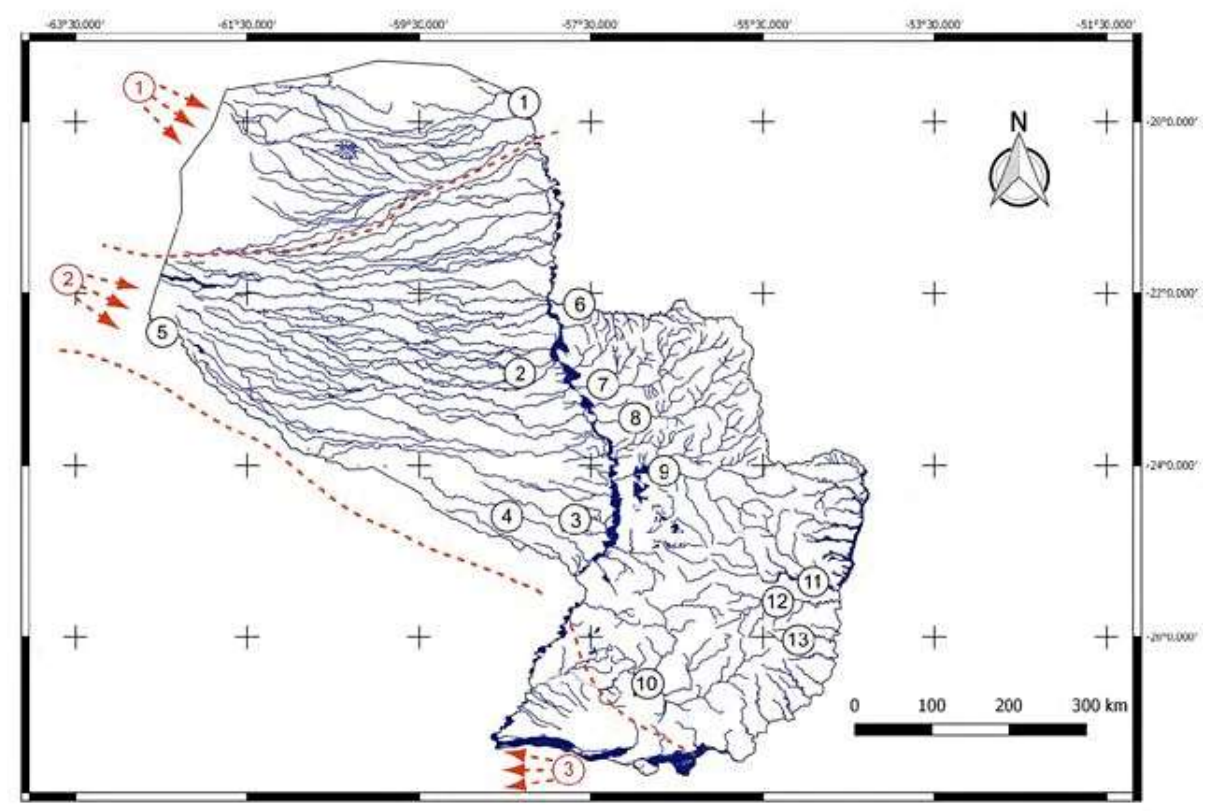

Nota. Tomado de Koerber, Vera-Alcaraz, y Reis (2017) 
El país se encuentra dividido en dos regiones por el Río Paraguay: a su margen derecha, la Región Occidental o Chaco; y a su margen izquierda, la Región Oriental. La población se encuentra distribuida en ambas a razón de 213.018 y 7.039 .654 habitantes, respectivamente (DGEEC 2020). En la Región Oriental, los sistemas de abastecimiento están alimentados por diversos acuíferos, como el Patiño en la región de los departamentos de Central y Paraguarí; y el Sistema Acuífero Guaraní (SAG), que abarca los departamentos de la Región Oriental del país, Alto Paraná, Amambay, Caaguazú, Caazapá, Canindeyú, Concepción, Guairá, Itapúa, Misiones, Ñeembucú y San Pedro. El mismo conforma una franja estimada de $200 \mathrm{~km}$ de ancho con dirección norte a sur que se extiende a lo largo de toda la Región Oriental y es, con excepción de Asunción y alrededores, la región más poblada del país, que alberga la mayor actividad económica dentro del territorio (Fariña 2007).

Desde el punto de vista geológico, el SAG se trata de un conjunto de areniscas depositadas entre 200 y 130 millones de años atrás que en total abarcan una superficie aproximada de $1.200 .000 \mathrm{~km}^{2}$, de los cuales a Paraguay le corresponden $71.700 \mathrm{~km}^{2}$ (Galdiano Gonçales et al. 2007). El acuífero se denomina sistema porque está conformado por varias unidades litoestratigráficas distintas que, desde la perspectiva hidrogeológica, interactúan entre sí y, por lo tanto, deben estudiarse en su conjunto (Ecosistema Urbano 2015a). El nombre “Guaraní" fue adoptado para unificar las distintas nomenclaturas utilizadas en los países para identificar la misma formación (Schmidt 2007): en Paraguay se conoce con el nombre de Alto Paraná, Misiones, y Grupo Independencia; en Brasil, como Serra Geral y Areniscas, Botucatú, Piramboia, y Grupo Passa Dois; en Argentina y Uruguay, como Tacuarembó, Buena Vista, y Grupo Melo; además de Serra Geral y Solari en Argentina; y Arapey y Areniscas en Uruguay (Jackson, Sangam, y Lafleur 2008).

La extracción total en la Región Oriental suma unos 173 millones de $\mathrm{m}^{3}$ por año, considerando un consumo de 150 litros/per cápita/día (Schmidt 2007). El consumo diario per cápita está entre los 150 y 200 litros (Herken y Ramírez 2015), al menos en el departamento de Central. La cantidad de pozos construidos se estiman alrededor de 1.400 unidades, en razón de 1.000 de estas correspondientes a organismos estatales ${ }^{1}$ y 400 pozos a entes privados o particulares ${ }^{2}$ (Larroza, Fariña, y Paredes 2001). Sin embargo, es muy

\footnotetext{
${ }^{1}$ Correspondientes al Servicio Nacional de Saneamiento Ambiental (SENASA) del Ministerio de Salud Pública y Bienestar Social (MSPyBS), Entidades Binacionales, Gobernaciones y Municipalidades

${ }^{2}$ Aguaterías Privadas y Comisiones Vecinales
} 
probable que esta cifra esté sub dimensionada, pues solo en el departamento de Itapúa existen 330 sistemas de provisión de agua potable que emplean fuentes subterráneas, de acuerdo a los registros del Ente Regulador de Servicios Sanitarios (2016). Cabe destacar que la Región Oriental del Paraguay está constituida por 14 de los 17 departamentos en los que se divide políticamente el país, de los cuales 11 se encuentran sobre el SAG.

\subsection{Vulnerabilidad a la contaminación de aguas subterráneas}

La vulnerabilidad de un acuífero a la contaminación se define como la sensibilidad del acuífero para ser adversamente afectado por una carga contaminante impuesta (Foster y Hirata 1991). Es decir que las características intrínsecas de los estratos que separan el sector de acuífero saturado de la superficie del terreno determinan la sensibilidad del acuífero de verse afectado por una carga contaminante aplicada en la superficie (Foster 1987). Considerando esto, la vulnerabilidad es función de la accesibilidad hidráulica a la penetración de un contaminante a la zona saturada, y de la capacidad de atenuación de los estratos sobre yacientes a la zona saturada, ya sea por fenómenos de retención físicoquímica o reacción con los contaminantes (Oleaga, Pacheco, y Feller 2009). Entonces, la vulnerabilidad intrínseca es aquella derivada de las características propias del acuífero y su entorno, sin considerar la acción de los contaminantes; mientras que la vulnerabilidad específica sí toma en cuenta la incidencia de sustancias contaminantes (Auge 2007).

Ciertos estudios previos establecieron que "el mapeo de la vulnerabilidad a la contaminación de un acuífero es normalmente el primer paso para la estimación del riesgo de contaminación del agua subterránea y protección de la calidad” (Oleaga et al. 2009). Existen diversas metodologías para la valoración de la vulnerabilidad de un acuífero a la contaminación, de los cuales la mayoría lo hace en función de las características intrínsecas del mismo. Los métodos más usados son DRASTIC, SINTACS, GOD y AVI; además, se encuentran disponibles otros como EPIK, EKv, $\triangle \mathrm{hT}$, y BGR, cada uno con sus particularidades.

Los métodos GOD y DRASTIC son los más comúnmente empleados. La aplicación de ambos se considera sencilla y depende de los datos disponibles (Auge 2007). En el caso del GOD se necesitan datos relativos al tipo de acuífero (G), litología de la zona no saturada (O) y profundidad del agua subterránea (D); mientras que para el DRASTIC, los datos hacen referencia a profundidad hasta el agua subterránea (D), recarga $(R)$, medio acuífero (A), medio del suelo (S), topografía (T), impacto de la zona vadosa (I), y 
conductividad del acuífero (C) (Foster et al. 2002; Foster y Hirata 1991; Musálem et al. 2015). En ambos casos, los resultados de vulnerabilidad en muchas áreas coinciden relativamente bien con estudios y mediciones de campo, y los mapas resultantes otorgan uma primera indicación general del peligro potencial de contaminación como herramienta de apoyo a los tomadores de decisión para la reglamentación, planificación y desarrollo del recurso (Oleaga et al. 2009). La diferencia radica en la disponibilidad de los datos, ya que GOD se emplea cuando éstos son escasos, no son fiables o no cubren la totalidad del territorio que se estudia (Palomino 2013); y DRASTIC emplea parámetros hidrológicos que caracterizan el destino y transporte de contaminantes y procesos de atenuación que actúan en la zona no saturada y en el agua subterránea en sí misma (Báez, Villalba, y Nogues 2014).

Entonces, la contaminación de acuíferos ocurre cuando las cargas contaminantes procedentes de actividades capaces de provocar riesgos significativos, como actividad agrícola, falta de saneamiento, industrias y otras, exceden la capacidad natural de atenuación del suelo y de los estratos subyacentes (Foster et al. 2002). Los contaminantes presentes en la superficie son transportados por el agua hasta el acuífero (Sánchez San Román 2017); no obstante, cuando éstos atraviesan la zona no saturada o vadosa, sufren procesos de atenuación natural debido a la degradación bioquímica, reacciones químicas o absorción por minerales arcillosos y/o materia orgánica (González Herrera, Rodríguez Castillo, y Coronado Peraza 2007). Sin embargo, la lenta circulación del agua subterránea, la degradación natural y las características propias del contaminante hacen que puedan transcurrir años antes que el impacto del mismo se haga evidente o sea detectado en pozos de extracción o monitoreo (Smith et al. 2016). Pero el problema real es que una vez el deterioro del agua subterránea se hace evidente, ya se encuentran afectados grandes volúmenes del acuífero (Foster et al. 2002; Mateos Ruiz et al. 2009).

\subsection{Provisión de agua en Paraguay}

Paraguay tiene una amplia disponibilidad de recursos hídricos por habitante. A pesar de ello, el $80 \%$ del abastecimiento público de agua en las comunidades del interior proviene de aguas subterráneas (PNUD 2006). Esta preferencia está dada por la contaminación cada vez más creciente de los cursos de agua superficiales, por la facilidad de acceso en zonas rurales, la disponibilidad relativamente permanente, y la calidad predominantemente satisfactoria (Larroza et al. 2001). 
La legislación paraguaya clasifica la provisión de agua de acuerdo a la población abastecida: a la Empresa de Servicios Sanitarios del Paraguay (ESSAP) le corresponden las localidades con más de 10.000 habitantes; mientras que al Servicio Nacional de Saneamiento Ambiental (SENASA), dependiente del Ministerio de Salud Pública y Bienestar Social (MSPyBS), aquellas con menos de 10.000 habitantes (Congreso de la Nación Paraguaya 2000). Otros actores involucrados son el Poder Ejecutivo, como titular del servicio; el Ministerio de Obras Públicas y Comunicaciones (MOPC), como rector de las políticas del sector; y la Dirección de Agua Potable y Saneamiento (DAPSAN), dependiente del mismo ministerio, como órgano técnico; y el Ente Regulador de Servicios Sanitarios (ERSSAN), como regulador, supervisor y administrador del sector (Ministerio de Obras Públicas y Comunicaciones y Dirección de Agua Potable y Saneamiento 2018; Ruiz 2020).

A grandes rasgos, se puede hacer una clasificación de los prestadores de acuerdo al tipo de fuente de agua a distribuir por las redes de tuberías: aquellos que emplean aguas superficiales, como ESSAP; y los que emplean aguas subterráneas. En este segundo grupo se encuentran varias organizaciones, como las Juntas de Saneamiento, una modalidad de gestión en la que las comunidades reciben los sistemas de provisión de parte de SENASA para encargarse de su operación, administración y mantenimiento; las Comisiones Vecinales, que son sistemas privados que se encuentran en camino de formalización para convertirse en Juntas de Saneamiento; los Prestadores Privados o Aguaterías, una iniciativa privada comunitaria cuya regulación está a cargo de ERSSAN; y otros proveedores, como Entidades Binacionales (Itaipú y Yacyretá), Gobernaciones Departamentales, y Cooperativas (Pacheco Figueredo, Servín Nasich, Servín Santa Cruz, et al. 2019; Velázquez Haurón, Pacheco Figueredo, et al. 2019).

En cuanto a los prestadores del servicio de distribución de agua, DAPSAN contabiliza 5.000 prestadores, de los cuales el 24,6\% corresponde a ESSAP; el 33,4\% a Juntas de Saneamiento; el 8,5\% a Comisiones Vecinales; el 9,2\% a Prestadores Privados; y el 2,5\% a otros (Ruiz 2020). Así, en 2020, se observa una importante cobertura de los servicios de agua mejorada a nivel país, alcanzando el 89,9\% en general, con una brecha entre el área urbana $(92,4 \%)$ y rural $(85,6 \%)$ (PNUD 2021). En general, el 80\% de las ciudades del interior del país emplean aguas subterráneas, mientras que en la capital, Asunción, se 
emplean mayormente aguas superficiales (Pacheco Figueredo, Servín Nasich, Velázquez Haurón, et al. 2019).

\subsection{La provisión de agua en el distrito de Encarnación}

Encarnación es la capital del departamento de Itapúa y la tercera más poblada de Paraguay, luego de Asunción y Ciudad del Este (Ecosistema Urbano 2015b). En los últimos 20 años la ciudad se ha visto afectada por el emprendimiento hidroeléctrico Yacyretá, lo que ha ocasionado cambios en la estructura social, territorial y ambiental como consecuencia de las obras ejecutadas para elevar el nivel del embalse de cota 76 a 83 msnm (Servín Nasich et al. 2017). A pesar de los impactos negativos, esto ha significado una serie de obras compensatorias y complementarias que trajeron diversas mejoras, especialmente en cuanto a los servicios sanitarios de la ciudad. Entre ellos se pueden mencionar la clausura del vertedero municipal y construcción de un relleno sanitario, ampliación de las distintas redes de provisión de agua, y la ampliación de la cobertura del alcantarillado sanitario hasta el alcanzar el 92\% del área urbana, con su correspondiente planta de tratamiento (Pacheco Figueredo, Servín Nasich, Velázquez Haurón, et al. 2019; Velázquez Haurón et al. 2017). Además, la construcción de tres playas artificiales, la Defensa Costera, la réplica de la Estación de Tren y el Centro de Convenciones, los cuales han aumentado significativamente el atractivo turístico de la ciudad (Servín Nasich 2014).

En cuanto al departamento de Itapúa, el servicio es proveído principalmente por Juntas de Saneamiento (23,8\%), Comisiones Vecinales (6\%) y Aguaterías Privadas (2,2\%), contabilizando un 30,9\% de la población servida por aguas subterráneas; a diferencia del 4,44\% de la población servida por ESSAP (Muñoz 2015).

Respecto al distrito de Encarnación, la situación del servicio es bastante particular: si bien el 83,63\% de la población tiene acceso a agua potable (Barrios 2016), un porcentaje similar al ámbito nacional, la proporción de uso de aguas superficiales y subterráneas es diferente al nivel departamental y nacional. Así, del total de la población servida, el 39,2\% recibe el servicio de ESSAP; y el 44,4\% de prestadores que emplean aguas subterráneas, como Juntas de Saneamiento, Comisiones Vecinales, Aguaterías Privadas y Otros (Barrios 2016; Pacheco Figueredo, Servín Nasich, Velázquez Haurón, et al. 2019). Esto se debe a las ventajas históricas del distrito por su calidad de capital departamental, además de las consecuencias positivas de las obras de compensación del emprendimiento 
hidroeléctrico Yacyretá. Por otra parte, resulta llamativo que un 17,10\% de la población indica que consume agua mineral para beber (Velázquez Haurón, Servín Nasich, et al. 2019), siendo que la principal fuente de estas el acuífero Guaraní.

\subsection{Vulnerabilidad del agua subterránea en las cuencas de los arroyos Quiteria y}

\section{Mbói Caé}

El distrito de Encarnación se encuentra asentado en el centro de las cuencas de los arroyos Quiteria y Mbói Caé, cuencas compartidas con los distritos de Fram, La Paz, Jesús, Trinidad, Nueva Alborada, Cambyretá, San Juan del Paraná y Capitán Miranda (Ecosistema Urbano 2015a; Musálem Castillejos 2008a, 2008b). En total cubren una superficie de 63.914 hectáreas, distribuidas en 35.273 para la cuenca del Quiteria y 28.641 para la cuenca del Mbói Caé (Ecosistema Urbano 2015a).

\section{Figura 2}

Distritos que componen las cuencas de los arroyos Quiteria y Mbói Caé

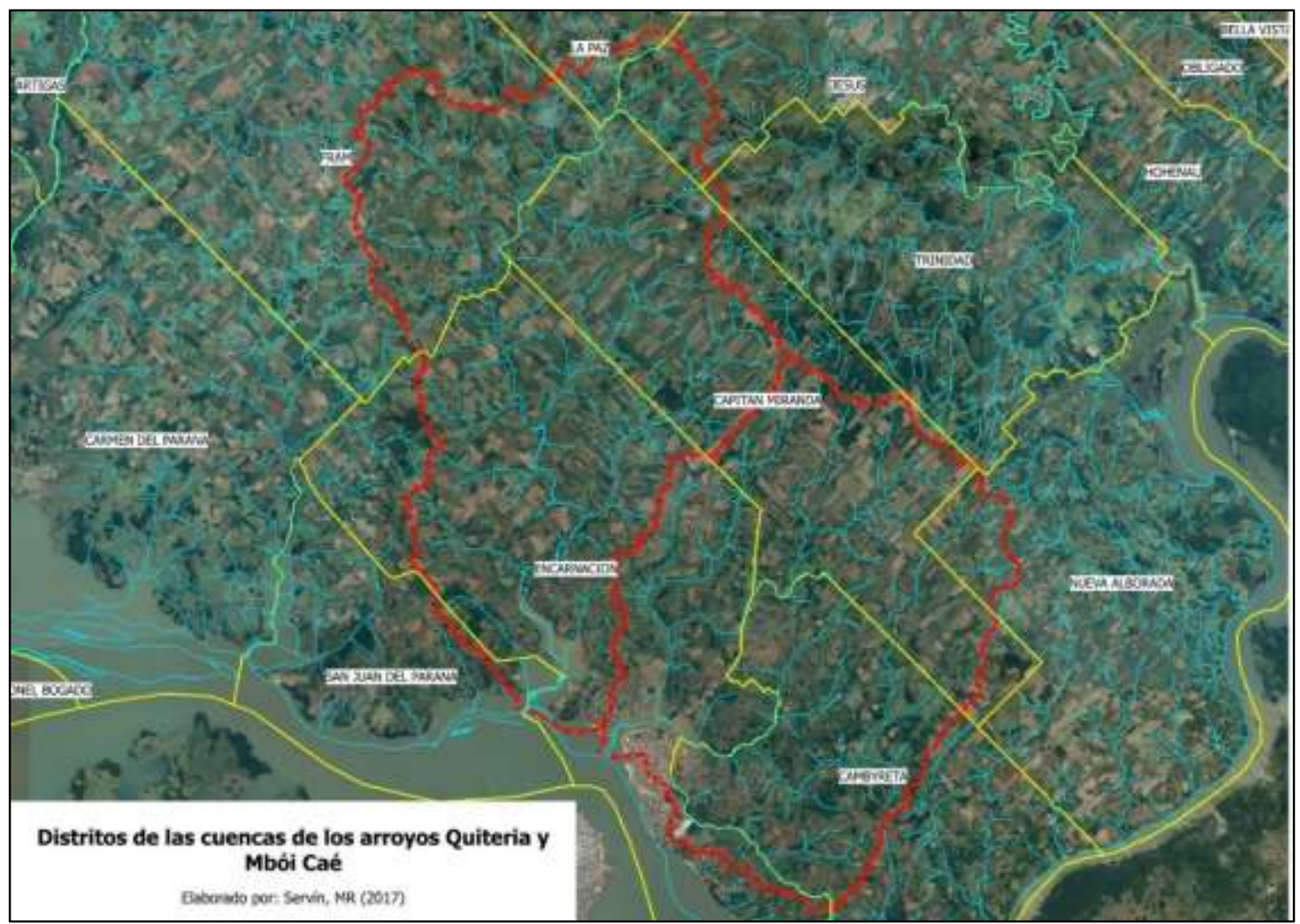

Nota. Extraído de Pacheco Figueredo, Servín Nasich, Servín Santa Cruz, et al. (2019)

En 2015, en estas cuencas se llevó a cabo un mapeo de la vulnerabilidad del agua subterránea, a través de los modelos DRASTIC y GOD (Musálem et al. 2015). En general, los autores encontraron que la vulnerabilidad del acuífero a la contaminación 
puede describirse como alta. Además, los valores de mayor vulnerabilidad se encontraron hacia las partes más bajas de la zona de estudio, coincidiendo con el área de mayor concentración urbana. Esto puede verse en la Figura 3:

\section{Figura 3}

Ubicación de las categorías de vulnerabilidad del agua subterránea a la contaminación en el área de las cuencas de los arroyos Quiteria y Mbói Caé, según modelo DRASTIC
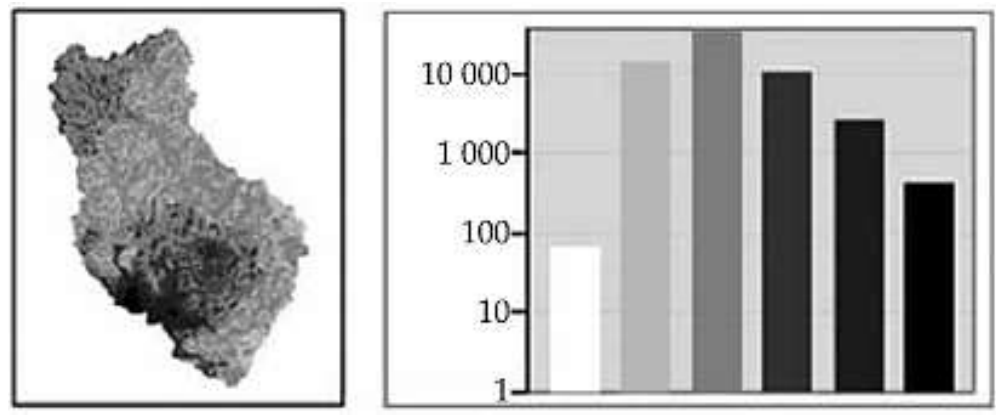

DRASTIC

Nota. Extraído de Musálem et al. (2015)

En cuanto a las aguas superficiales, Pacheco Figueredo, Servín Nasich, Servín Santa Cruz, et al. (2019) llegaron a conclusiones similares a través de mediciones de parámetros físico-químicos y bacteriológicos, así como de diversos índices de calidad de agua, realizados en 2018 en la misma área. Las concentraciones de nitrógeno amoniacal (N$\mathrm{NH}_{4}$ ), coliformes fecales y fósforo total son los principales parámetros que se encuentran fuera de los rangos permitidos por la autoridad ambiental. Respecto a los índices aplicados $\left(\mathrm{INSF}^{3}, \mathrm{DMR}^{4}\right.$, Dinius $\left.{ }^{5}, \mathrm{ISQA}^{6}, \mathrm{e} \mathrm{ICOs}^{7}\right)$, los peores resultados se observan también en la cuenca baja de los arroyos Quiteria y Mbói Caé. Como respuesta, dichos autores han propuesto en 2019 un Plan de Gestión Integrada de Recursos Hídricos, que luego fue declarado de interés por la Junta Municipal de la ciudad en 2021.

Debido a la importancia de las aguas subterráneas en el sistema de provisión de agua en el distrito de Encarnación, para este trabajo analizó la relación entre la vulnerabilidad a la contaminación de las aguas subterráneas, según el método DRASTIC, de acuerdo a los resultados de Musálem et al. (2015), y la ubicación de los pozos de abastecimiento comunitario. A través de este análisis, se plantean los siguientes objetivos: (a) distinguir

\footnotetext{
3 Índice de la National Sanitatio Foundation (NSF) de Estados Unidos

4 Índice del río Des Moines de Estados Unidos

5 Índice Dinius, de Brown y McLeland (1973), para diversos usos

6 Índice Simplificado de Calidad de Agua, de de Bustamante (1989)

7 Índices de Contaminación, de Ramírez, Restrepo, y Cardeñosa (1999) y Ramírez Silva, Restrepo, y Viña (1997)
} 
los pozos de captación de aguas subterráneas para distribución en redes de tubería que se encuentran ubicados en las zonas de mayor vulnerabilidad a la contaminación; y, (b) estimar el volumen de agua distribuida en estas redes que podría verse comprometido por la vulnerabilidad propia del acuífero. De esta manera, podrán identificar los puntos y áreas que merecen mayor consideración en fases posteriores.

\section{ESTRATEGIAS METODOLÓGICAS}

El estudio fue llevado a cabo en el área de las cuencas de los arroyos Quiteria y Mbói Caé, ubicadas al sur de la República del Paraguay, en el departamento de Itapúa. Además, estuvo enfocado en el distrito de Encarnación, por su carácter de capital departamental, ya que concentra una mayor población en relación a los demás distritos, con 138.592 habitantes, según la proyección al 2021 (Instituto Nacional de Estadística 2021). El mismo se encuentra a aproximadamente $400 \mathrm{~km}$ al sur de la ciudad capital, Asunción. La ubicación del departamento en el contexto nacional y del distrito en el departamento puede observarse en la Figura 4:

\section{Figura 4}

Ubicación del área de estudio
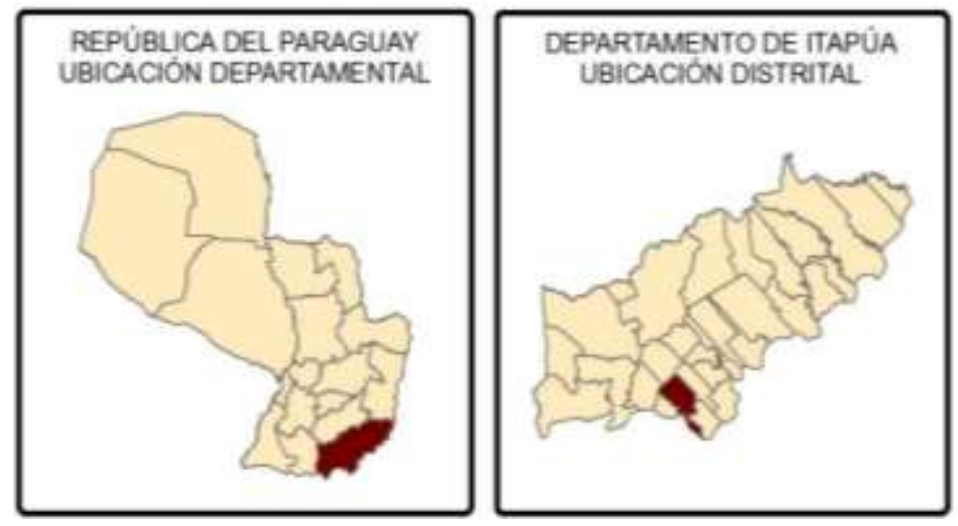

Nota. Extraído de Servín Nasich (2014)

El enfoque de la investigación es cuantitativo, descriptivo, no experimental y transversal.

Es cuantitativo porque busca medir y estimar magnitudes del fenómeno de estudio (Hernández Sampieri y Mendoza Torres 2018); descriptivo, porque señala sus características más importantes (Sánchez Carlessi, Reyes Romero, y Mejía Sáenz 2018) dentro de un contexto particular (Hurtado de Barrera 2000); no experimental, porque no hay manipulación de variables (Ortiz Uribe 2004); y transversal, debido a que los datos fueron recopilados en un momento único (Hernández Sampieri, Fernández Collado, y Baptista Lucio 2014). Los resultados aquí presentados forman parte de la investigación 
realizada en el marco del Doctorado en Gestión Ambiental de la Escuela de Posgrado de la Universidad Nacional de Itapúa (Paraguay).

Todo el departamento de Itapúa se encuentra asentado sobre el SAG (Ecosistema Urbano 2015a). De acuerdo a PNUD (2006), el acuífero que se utiliza para extracción de aguas subterráneas es la formación Misiones y, como explica Schmidt (2007), esta forma parte del Sistema Acuífero Guaraní (SAG). Aun así, el nivel de explotación actual resulta muy difícil de calcular, debido a la falta de información al respecto, particularmente sobre la intensidad y finalidad de usos. Es importante mencionar que el área de estudio se encuentra sobre la zona de recarga del mencionado acuífero.

Los datos empleados para el alcance de ambos objetivos provienen de fuentes secundarias. Primero, se realizó un cruzamiento entre los resultados obtenidos por Musálem et al. (2015) a través de los modelos DRASTIC y GOD para determinar la vulnerabilidad del agua subterránea a la contaminación, con la ubicación de los pozos de abastecimiento comunitario, de acuerdo a datos proveídos por la Oficina Regional del Ente Regulador de Servicios Sanitarios (ERSSAN), al 2016. Y, segundo, se calcularon las extracciones de agua subterránea para abastecimiento comunitario en el distrito de Encarnación a través de la fórmula propuesta por Vassolo (2007), conforme la siguiente ecuación:

\section{Población $\times$ consumo $=$ extracciones}

En este contexto, el consumo se mide en $\mathrm{m}^{3}$ por 365 días en un año; dando como resultado el valor de las extracciones en $\mathrm{m}^{3} / a n ̃ o$. Así, por conversión simple, puede hallarse la equivalencia en $\mathrm{km}^{3} /$ año.

Los datos de población fueron obtenidos de las proyecciones de población para el departamento de Itapúa para el año 2016 de la Dirección General de Estadísticas, Encuestas y Censos (DGEEC), hoy Instituto Nacional de Estadística (INE); mientras que el consumo de agua fue considerado de 175 litros diarios por persona, calculados en base al promedio de consumo del departamento Central, que considera entre 150 y 200 litros (Herken y Ramírez 2015). Por medio de este análisis se puede estimar el volumen de agua distribuida en redes de tubería en estos sistemas comunitarios que podría verse 
comprometido por la combinación entre la vulnerabilidad propia del acuífero y la ubicación de los pozos de captación.

Existe una falta de registros oficiales de los pozos extractivos, tanto de los sistemas en red como de los pozos artesianos para abastecimiento propio. Datos proveídos por ERSSAN (Barrios 2016) mencionan que, sólo en Itapúa, hay 330 sistemas que se proveen de aguas subterráneas, de los cuales 52 corresponden a Encarnación. Entonces, puede decirse que los registros están sub dimensionados. Entonces, tomando en cuenta la disponibilidad de datos oficiales, para fines de este estudio solo se consideran las perforaciones del distrito de Encarnación cuyo objetivo sea la captación de aguas subterráneas para abastecimiento comunitario.

\section{RESULTADOS Y DISCUSIÓN}

\subsection{Pozos de captación ubicados en zonas vulnerables a la contaminación}

A continuación, se presentan los resultados de la combinación de los valores de vulnerabilidad a la contaminación de las aguas subterráneas en las cuencas de los arroyos Quiteria y Mbói Caé puede, según el método DRASTIC, junto con la ubicación los pozos de captación de los sistemas de provisión de agua por redes de tubería correspondientes al distrito de Encarnación:

\section{Figura 5}

Vulnerabilidad a la contaminación de las aguas subterráneas en las cuencas estudiadas

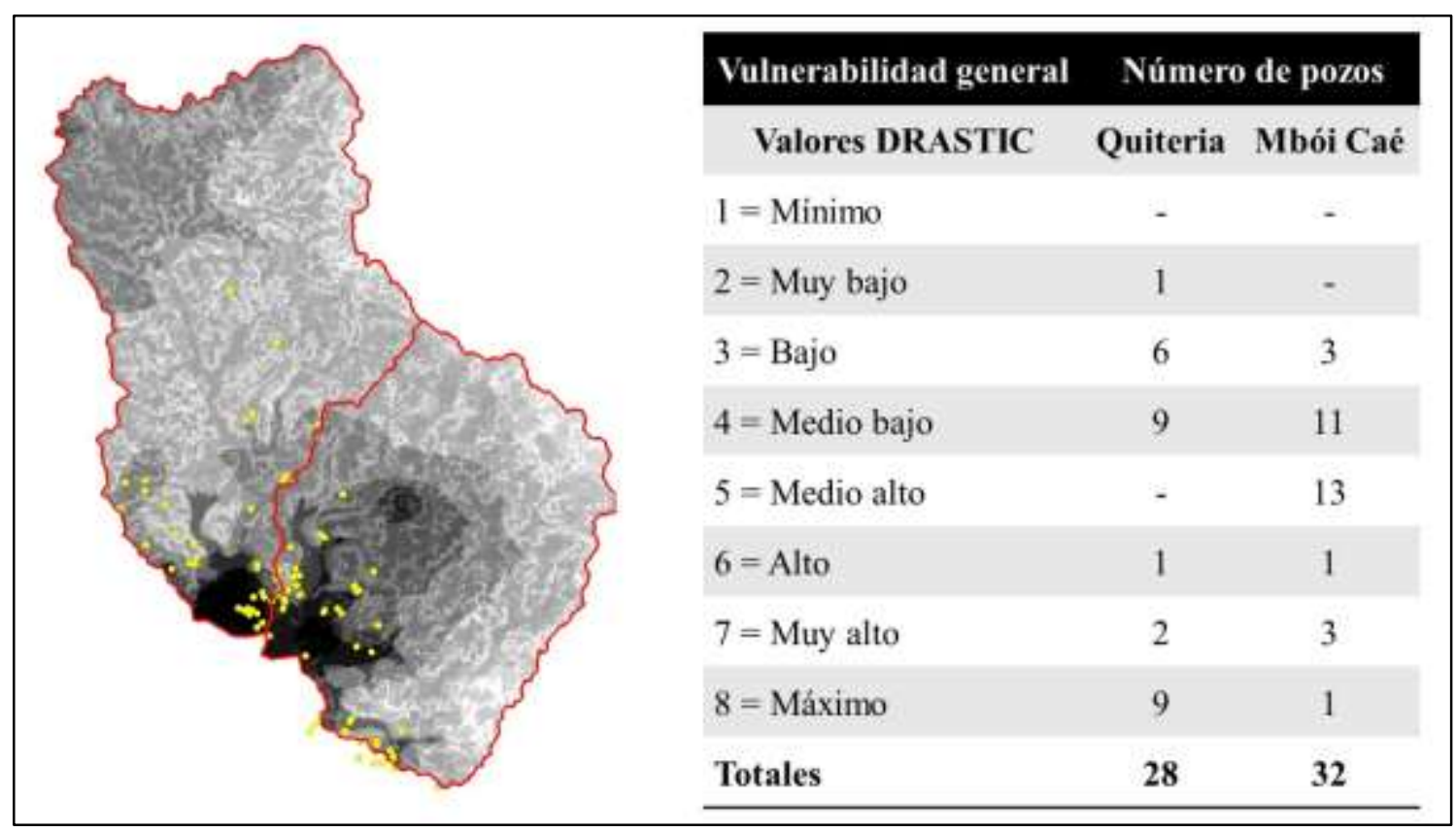

Nota. Elaboración propia a partir de Musálem et al. (2015) y Barrios (2016) 
En amarillo se observan los pozos de captación y, en rojo, los límites de las cuencas en estudio. En la parte superior se encuentra la cuenca del arroyo Quiteria y, en la parte inferior, la del arroyo Mbói Caé. En ellas se ubicaron 28 y 32 pozos de captación, respectivamente, contabilizándose un total de 60 sistemas comunitarios, hasta 2016.

Considerando las distintas categorías de vulnerabilidad establecidas para el método DRASTIC, los pozos de captación en ambas cuencas se distribuyen de la siguiente manera:

\section{Figura 6}

Número de pozos por categoría de vulnerabilidad DRASTIC, según cuenca

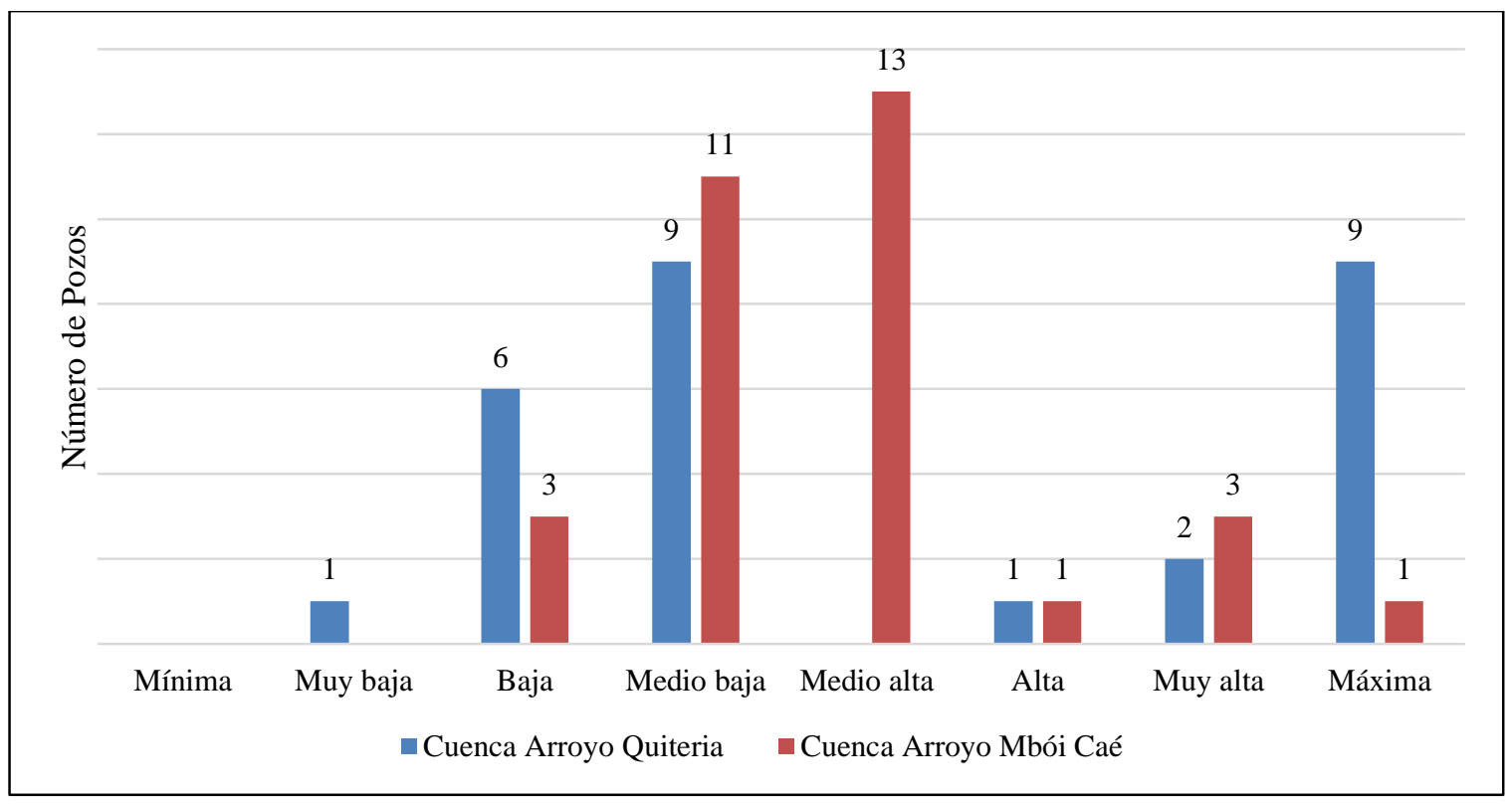

Nota. Elaboración propia

En cuanto al arroyo Quiteria, la mayor parte de los pozos de captación $(53,57 \%)$ se encuentran ubicados sobre áreas de vulnerabilidad a la contaminación consideradas como bajas a medio altas. Le sigue un segundo grupo de pozos ubicados en áreas de muy alta a máxima vulnerabilidad (39,28\%). Respecto al arroyo Mbói Caé, la mayor parte de los pozos $(75 \%)$ se encuentra ubicado en áreas consideradas de vulnerabilidad medio baja a medio alta.

\subsection{Volumen de extracciones de agua subterránea para abastecimiento comunitario}

Los prestadores y las fuentes de agua de las que se sirve la población del departamento de Itapúa y del distrito de Encarnación, se distribuyen de la siguiente manera: 


\section{Tabla 1}

Cobertura del servicio de agua potable con redes de tubería en el departamento de Itapúa y el distrito de Encarnación (Paraguay)

\begin{tabular}{lcccc}
\hline & \multicolumn{2}{c}{ Sistemas superficiales } & \multicolumn{2}{c}{ Sistemas subterráneos } \\
\cline { 2 - 5 } & Itapúa & Encarnación & Itapúa & Encarnación \\
\cline { 2 - 5 } $\mathbf{N}^{\circ}$ de sistemas & 3 & 2 & 330 & 52 \\
$\mathbf{N}^{\circ}$ de conexiones & 13.681 & 11.618 & 64.369 & 13.186 \\
$\begin{array}{l}\text { Población } \\
\text { conectada }\end{array}$ & 58.828 & 49.957 & 298.939 & 56.700 \\
Cobertura (\%) & 10,60 & 39,71 & 51,2 & 44,46 \\
\hline
\end{tabular}

Nota. Elaboración propia a partir de datos proveídos por ERSSAN

Según datos oficiales del Ente Regulador de Servicios Sanitarios (ERSSAN), el 83,63\% de la población encarnacena tiene acceso a agua potable, correspondiendo a una población de 106.650 habitantes, al 2016. El 16,67\% restante o no tiene acceso o no se tiene registro al respecto, lo cual podría indicar que son conexiones clandestinas o tienen un sistema de auto abastecimiento, probablemente a través de pozos artesianos.

A partir de estos datos, si se considera la población del distrito de Encarnación servida por sistemas que emplean aguas subterráneas, es decir, 56.700 habitantes; y un consumo de 180 litros diarios o $0,18 \mathrm{~m}^{3}$ por día, las extracciones para uso doméstico resultan en 3.725.190 $\mathrm{m}^{3} /$ año. Entonces, las extracciones por sistemas de provisión de agua que emplean fuentes subterráneas en Encarnación contabilizan un total de 0,004 km³/año.

Teniendo en cuenta que el proyecto SAG valora, en general, las extracciones de las principales ciudades ubicadas sobre el área de este acuífero como despreciables, podría decirse que las extracciones del distrito de Encarnación $(0,004$ km³/año), en relación a la recarga total del SAG $\left(10,39 \mathrm{~km}^{3} / \mathrm{año}^{8}\right)$, son prácticamente insignificantes. Aun así, considerando la alta dependencia de las aguas subterráneas por parte de la población local, el volumen de las extracciones podría afectar, al menos, a un total de 56.700 personas. Esto, sin tomar en cuenta otros pozos de abastecimiento privado, así como las extracciones comerciales y el aumento de población en temporadas turísticas, por lo que esta cifra podría ser aún mucho mayor.

\footnotetext{
${ }^{8}$ Este valor corresponde a la recarga total del SAG en Brasil, Argentina, Paraguay y Uruguay. La recarga en Paraguay es de $6,5 \mathrm{~km}^{3} /$ año, aproximadamente.
} 


\section{CONSIDERACIONES FINALES}

Una vez establecido el panorama general del agua en Paraguay y el departamento de Itapúa sobre el Sistema Acuífero Guaraní (SAG), este estudio analizó la relación entre la vulnerabilidad a la contaminación de las aguas subterráneas según los resultados de Musálem et al (2015) y la ubicación de los pozos de abastecimiento comunitario en el distrito de Encarnación. Así, primero, se distinguieron los pozos de captación de aguas subterráneas para distribución en redes de tubería que se encuentran ubicados en las zonas de mayor vulnerabilidad a la contaminación; y, segundo, se estimó el volumen de agua distribuida en estas redes que podría verse comprometido por la vulnerabilidad propia del acuífero.

En cuanto al primer resultado, gran parte de la población del distrito de Encarnación se encuentra asentada en las áreas más vulnerables a la contaminación, coincidiendo con la zona urbana. Además, la mayor parte de los pozos de captación de aguas subterráneas para distribución en redes de tubería se encuentran ubicados en áreas con vulnerabilidad entre medio baja y medio alta, y un grupo importante con vulnerabilidad máxima.

Así, se encontraron valores de mayor vulnerabilidad hacia las partes más bajas de ambas cuencas, coincidiendo con el área más urbanizada del distrito de Encarnación. Esto demuestra que la preocupación por la contaminación del acuífero en el mediano plazo se debe considerar para instrumentar programas posibles dedicados a la minimización de contaminantes. De esta manera, los resultados de la investigación sugieren la necesidad de identificar las fuentes de contaminación en estas áreas como posibles amenazas al acuífero, así como verificar el cumplimiento de normativas relativas a la provisión de agua. Efectivamente, los mapas de evaluación de la vulnerabilidad del acuífero a la contaminación resultan una herramienta útil para iniciar un proceso de gestión ambiental. Respecto del segundo resultado, se pudo estimar el volumen de aguas subterráneas que son distribuidas en redes de tubería, por medio de sistemas comunitarios. Cabe destacar que estos pozos de captación no son los únicos que hacen uso de este recurso pero, dada la disponibilidad de datos, el cálculo de extracciones no considera otros pozos como aquellos de abastecimiento individual en viviendas y edificios, ni aquellos de extracción comercial, como las empresas de agua mineral e industrias locales. Por este motivo, resulta de suma importancia recabar estos datos, de manera a actualizar los resultados obtenidos, incluso, discriminándolos de acuerdo al tipo de uso. 
La alta dependencia de las aguas subterráneas, de casi la mitad de la población local, puede considerarse una señal de alerta ante el peligro potencial a la contaminación de estas fuentes de agua, dada la alta concentración de población y de actividades económicas de diversa índole que podrían afectarlas. Esto ya ha generado un impacto negativo sobre la calidad de las aguas superficiales y, aunque las aguas subterráneas cuentan con la protección de la capa de basalto de la Formación Misiones, al ser el distrito de Encarnación considerado como parte del área de recarga del SAG, este es un aspecto que no puede ser ignorado. Independientemente del contaminante a ser considerado, una vez identificado en los análisis de calidad de las aguas subterráneas, esto será indicador de que un gran volumen del sistema ha sido afectado.

Los resultados obtenidos en este estudio pueden considerarse el primer paso hacia una gestión sostenible del recurso subterráneo. Dadas las consecuencias potenciales de la contaminación, especialmente en cuanto a la salud pública, resulta de suma importancia definir perímetros de protección de pozos para las fuentes de captación de aguas subterráneas, así como estudiar el nivel de aplicación de las normativas vigentes. Además, es necesario hacer un inventario de contaminantes, de forma a identificar las fuentes puntuales y potenciales de contaminación, para lo cual se recomienda utilizar el método POSH propuesto por Foster et al. (2002). Esto podrá luego implementarse y monitorearse como parte de una estrategia de ordenamiento territorial, lo cual es responsabilidad del municipio local.

Agradecimientos: Los autores agradecen al Consejo Nacional de Ciencia y Tecnología (CONACYT) de Paraguay, por el financiamiento del programa de Doctorado en Gestión Ambiental de la Universidad Nacional de Itapúa; a Karim Musálem y Rafaela Laíno, por la provisión de datos DRASTIC; y a la Dirección Regional Itapúa del Ente Regulador de Servicios Sanitarios (ERSSAN), por la provisión de datos sobre sistemas de distribución de agua por redes de tuberías en Encarnación.

\section{LISTA DE REFERENCIAS}

Álvarez Enciso, María del Carmen. 2014. Disponibilidad hídrica del Paraguay. Asunción, Paraguay: Instituto Desarrollo.

Auge, Miguel. 2007. Vulnerabilidad de Acuíferos. Buenos Aires, Argentina: Instituto de Geología y Recursos Naturales. 
Báez, Liz, Cynthia Villalba, y Juan Pablo Nogues. 2014. «Estudio 2: Metodología». Acuífero Patiño. Estudio del Riesgo de Contaminación. Recuperado 18 de septiembre de 2021 (http://www.estudiopatino.pol.una.py/blog/?page_id=268).

Barrios, Pablo. 2016. «Provisión de datos sobre Juntas de Saneamiento de Encarnación». de Bustamante, Irene. 1989. «Aspectos metodológicos en estudios de calidad de agua». Henares (3):25-36.

Congreso de la Nación Paraguaya. 2000. «Ley N 1614 - General del Marco Regulatorio y Tarifario del Servicio Público de Provisión de Agua Potable y Alcantarillado Sanitario para la República del Paraguay».

Dirección General de Estadística, Encuestas y Censos. 2020. «DGEEC comparte datos de la población alusivos a la Paz del Chaco». Recuperado 19 de septiembre de 2021 (https://www.ine.gov.py/news/news-contenido.php?cod-news=472).

Ecosistema Urbano. 2015a. Medioambiente y Territorio. 2. Encarnación, Paraguay: Municipalidad de Encarnación.

Ecosistema Urbano. 2015b. Sociedad. 6. Encarnación, Paraguay: Municipalidad de Encarnación.

Fariña, Sandra. 2007. Geología e Hidrogeología. Asunción, Paraguay: SEAM - BGR.

Fernáncez Cirelli, Alicia, y Cecile du Mortier. 2005. «Evaluación de la condición del agua para consumo en Latinoamérica». en Posibilidades para la provisión de agua segura usando nuevas tecnologías. Solar Safe Water.

Fernández Colón, Gustavo. 2009. «La crisis del agua en América Latina». Estudios Culturales II(4):80-96.

Foster, Stephen. 1987. «Fundamental concepts in aquifer vulnerability, pollution risk and protection strategy». en Vulnerability of soil and groundwater to polluants. International conference. Nordwijk, The Netherlands.

Foster, Stephen, y Ricardo Hirata. 1991. Determinación del riesgo de contaminación de aguas subterráneas: una metodología basada en datos existentes. Lima, Perú: Organización Panamericana de la Salud.

Foster, Stephen S. D., Ricardo Hirata, Daniel Gomes, Monica D’Elia, y Marta Paris. 2002. Groundwater quality protection: a guide for water utilities, municipal authorities, and environment agencies. Washington, D.C: World Bank. 
da Franca Ribeiro dos Anjos, Nelson, Michela Miletto, María Concepción Donoso, Alice Aureli, Shammy Puri, Jac Van der Gun, Ofelia Tujchneider, y Alfonso Rivera. 2007. Programa Unesco/OEA ISARM Américas acuíferos transfronterizos de las Américas: Sistemas acuíferos transfronterizos en las américas, evaluación preliminar. Montevideo, Uruguay: Unesco.

Galdiano Gonçales, Valter, Mário Nascimento Souza Filho, Ivanir Borella Mariano, y Carlos Eduardo Quaglia Giampá. 2007. Manual de Perforación de Pozos Tubulares para Investigación y Captación de Aguas Subterráneas en el Sistema Acuífero Guaraní. Montevideo, Uruguay.

González Herrera, Roger, Ramiro Rodríguez Castillo, y Víctor Coronado Peraza. 2007. «Atenuación natural en el acuífero Yucateco». Revista Internacional de Contaminación Ambiental 23(1):5-15.

Herken, Verónica, y Juan Ramírez. 2015. «Guía de uso eficiente del agua».

Hernández Sampieri, Roberto, Carlos Fernández Collado, y Pilar Baptista Lucio. 2014. Metodología de la investigación. México: McGraw Hill Interamericana.

Hernández Sampieri, Roberto, y Christian Paulina Mendoza Torres. 2018. «Glosario». P. 21 en Metodología de la investigación: las rutas cuantitativa, cualitativa y mixta. Hurtado de Barrera, Jacqueline. 2000. Metodología de la Investigación Holística. 3. a ed. Caracas, Venezuela: Fundación Sypal.

Instituto Nacional de Estadística. 2021. Itapúa: proyecciones de población por sexo y edad 2021. Asunción, Paraguay: INE.

Jackson, Helen, Henri Sangam, y Adriana Lafleur. 2008. Hidrogeología local del área piloto Itapúa. Sistema Acuífero Guaraní. SNC Lavalin International.

Koerber, Stefan, Héctor S. Vera-Alcaraz, y Roberto E. Reis. 2017. «Checklist of the Fishes of Paraguay (CLOFPY)». Ichthyological Contributions of PecesCriollos 53:1-99.

Larroza, Fernando, Sandra Fariña, y José Luis Paredes. 2001. «Consideraciones preliminares del Acuífero Guaraní en el Paraguay Oriental». P. 10 en. Asunción, Paraguay.

Maboggiano. 2019. «Los 10 países con mayores reservas de agua dulce». Columnistas. $\begin{array}{lllll}\text { Recuperado } & 19 & \text { de } & \text { septiembre } & \text { de }\end{array}$ 
(https://www.cronista.com/columnistas/Los-10-paises-con-mayores-reservas-deagua-dulce-20191125-0037.html).

Mateos Ruiz, Juan Antonio, Juan María Fornés Azcoiti, Gerardo Ramos González, y Fermín Villarroya Gil. 2009. Las aguas subterráneas. Un recurso natural del subsuelo. Madrid: Instituto Geológico y Minero de España.

Ministerio de Obras Públicas y Comunicaciones, y Dirección de Agua Potable y Saneamiento. 2018. «Plan Nacional de Agua Potable y Saneamiento».

Monte Domecq, Roger. 2003. «Paraguay». en Inundações urbanas na América do Sul. Porto Alegre (RS): dos Autores.

Muñoz, Cristina. 2015.«Ponencia del Ente Regulador de Servicios Sanitarios del Paraguay (ERSSAN)». Presentado en Cobertura y calidad del agua potable en la ciudad de Encarnación, junio 26, Encarnación, Paraguay.

Musálem Castillejos, Karim. 2008a. Caracterización del arroyo Mbói Caé, Itapúa, Paraguay. Entidad Binacional Yacyretá.

Musálem Castillejos, Karim. 2008b. Caracterización del arroyo Quiteria, Itapúa, Paraguay. Entidad Binacional Yacyretá.

Musálem, Karim, Morag McDonald, Francisco Jiménez, y Rafaela Laino. 2015. «Groundwater Vulnerability Mapping in Two Watersheds Affected by Yacyreta Dam in Paraguay». Tecnología y Ciencias Del Agua 13.

Oleaga, Alejandro, Fernando Pacheco, y Moshe Feller. 2009. Determinación de perímetros de protección de pozos y vulnerabilidad y riesgo de contaminación de acuíferos. Vol. 6. Montevideo, Uruguay.

Ortiz Uribe, Frida Gisela. 2004. Diccionario de metodología de la investigación científica. México: Limusa.

Pacheco Figueredo, Viviana Marilyn, María Rosa Servín Nasich, Miguel Ángel Servín Santa Cruz, y Edith Jacqueline Velázquez Haurón. 2019. Plan de Gestión Integral de Recursos Hídricos para la ciudad de Encarnación. Resultados del Proyecto 14 INV 223. Encarnación, Paraguay: Universidad Nacional de Itapúa.

Pacheco Figueredo, Viviana Marilyn, María Rosa Servín Nasich, Edith Jacqueline Velázquez Haurón, y Miguel Ángel Servín Santa Cruz. 2019. «Acceso al agua potable y saneamiento: el caso de la ciudad de Encarnación - Paraguay». Pp. 7381 en Encarnación antes, durante y después de la suba del embalse: aspecto 
social, económico y ambiental. Encarnación, Paraguay: Universidad Nacional de Itapúa.

Palomino, O. 2013. «Análisis de riesgo de contaminación de las aguas subterráneas utilizando mapa de vulnerabilidad y peligro en el caso Acuífero Aguascoca». P. 6 en Memoria Segundo Encuentro de Investigadores Ambientales. Arequipa, Perú: Ministerio del Ambiente. Dirección General de Investigacón e Información Ambiental.

Programa de las Naciones Unidas para el Desarrollo. 2006. Usos y Gobernabilidad del Agua en Paraguay. Programa de las Naciones Unidas para el Desarrollo.

Programa de las Naciones Unidas para el Desarrollo. 2021. Segundo Informe Nacional Voluntario Paraguay 2021. Caminando juntos hacia un Paraguay más inclusivo, participativo y resiliente. Asunción, Paraguay: Comisión ODS Paraguay.

Ramírez, Nubia E., R. Restrepo, y M. Cardeñosa. 1999. «Índices de contaminación para caracterización de aguas continentales y vertimientos: Formulaciones». $C T \& F$ Ciencia, tecnología y futuro 1(5):89-99.

Ramírez Silva, Ana Beatriz, R. Restrepo, y G. Viña. 1997. «Cuatro índices de contaminación para caracterización de aguas continentales: Formulaciones y aplicación». CT\&F - Ciencia, tecnología y futuro 1(3):135-53.

Ruiz, María Celeste. 2020. El acceso al agua en América Latina y su consecuencia durante la pandemia. Universitát Politécnica de Catalunya.

Sánchez Carlessi, H. Hugo, Carlos Reyes Romero, y Katia Mejía Sáenz. 2018. Manual de términos en investigación científica, tecnológica y humanística. Perú: Universidad Ricardo Palma.

Sánchez San Román, F. Javier. 2017. Contaminación de las aguas subterráneas. Createspace Independent Pub.

Schmidt, Gerhard. 2007. Generalidades y Resumen. Asunción, Paraguay: SEAM - BGR. Servín Nasich, María Rosa. 2014. «Transformaciones territoriales urbanas de la ciudad de Encarnación, un análisis desde la afluencia turística». Maestría en Manejo de Recursos Naturales y Gestión Ambiental del Territorio, Universidad Nacional de Asunción, San Lorenzo, Paraguay.

Servín Nasich, María Rosa, Miguel Ángel Servín Santa Cruz, Edith Jacqueline Velázquez Haurón, y Viviana Marilyn Pacheco Figueredo. 2017. «Evolution water quality of 
urban streams of the Mbói Kaé basin with regard to the Yacyretá dam level». Pp. 17-25 en. Asunción, Paraguay.

Smith, Mark, Katharine Cross, Mary Paden, y Peter Laban, eds. 2016. Spring : managing groundwater sustainably. International Union for Conservation of Nature and Natural Resources.

Vassolo, Sara. 2007. Modelo de aguas subterráneas. Asunción, Paraguay: SEAM - BGR. Velázquez Haurón, Edith Jacqueline, Viviana Marilyn Pacheco Figueredo, María Rosa Servín Nasich, y Miguel Ángel Servín Santa Cruz. 2019. «La gobernabilidad y la gestión de cuencas». Pp. 161-75 en Conhecimento em rede: desenvolvimento, cooperação e integração regional em território de fronteira - Rede CIDIR: 10 anos. Rede CIDIR.

Velázquez Haurón, Edith Jacqueline, María Rosa Servín Nasich, Viviana Marilyn Pacheco Figueredo, y Miguel Ángel Servín Santa Cruz. 2019. ¿Cómo vivimos en Encarnacion? Perspectiva Ciudadana. Encarnación, Paraguay: Universidad Nacional de Itapúa.

Velázquez Haurón, Jacqueline, Viviana Marilyn Pacheco Figueredo, María Rosa Servín Nasich, y Miguel Ángel Servín Santa Cruz. 2017. «Encarnación, antes, durante y después de la suba del embalse: aspecto social, económico y ambiental». La Saeta Universitaria Académica y de Investigación 6(1).

Wilkinson, John. 2010. Agua y Tierra en América Latina: Estrategias Globales y Políticas. Plataforma Democrática. 